\title{
Dystrophic calcification after palliative chemo- therapy in a patient with renal cell carcinoma
}

\author{
Do Hyoung Lim, Soon Il Lee, and Keon Woo Park
}

Division of Hematology and Oncology, Department of Internal Medicine, Dankook University Hospital, Cheonan, Korea
Received: September 22, 2015 Revised : September 23, 2015 Accepted: October 23, 2015

\section{Correspondence to}

Soon Il Lee, M.D.

Tel: +82-41-550-3922

Fax: +82-41-550-7058

E-mail: avnrt@hanmail.net
A 71-year-old woman presented with a 2-week history of abdominal and back pain. She had undergone right radical nephrectomy about 2 years ago due to renal cell carcinoma. Computed tomography (CT) scans of the abdominopelvic area revealed enhancing masses with prominent necrotic change in the aortocaval and left paraaortic spaces, which were about 7.4 and $1.5 \mathrm{~cm}$ in size, respectively (Fig. 1). After full evaluation, she was diagnosed with recurrent renal cell carcinoma with multiple lymph node metastases and she received palliative chemotherapy with weekly temsirolimus $(25 \mathrm{mg}$ intravenous injection over 1 hour). The initial treatment plan was that chemotherapy would be continued until disease progression or development of an unacceptable side effect, and she received repeat chemotherapy due to best response with partial remission without any serious side effects. At 80
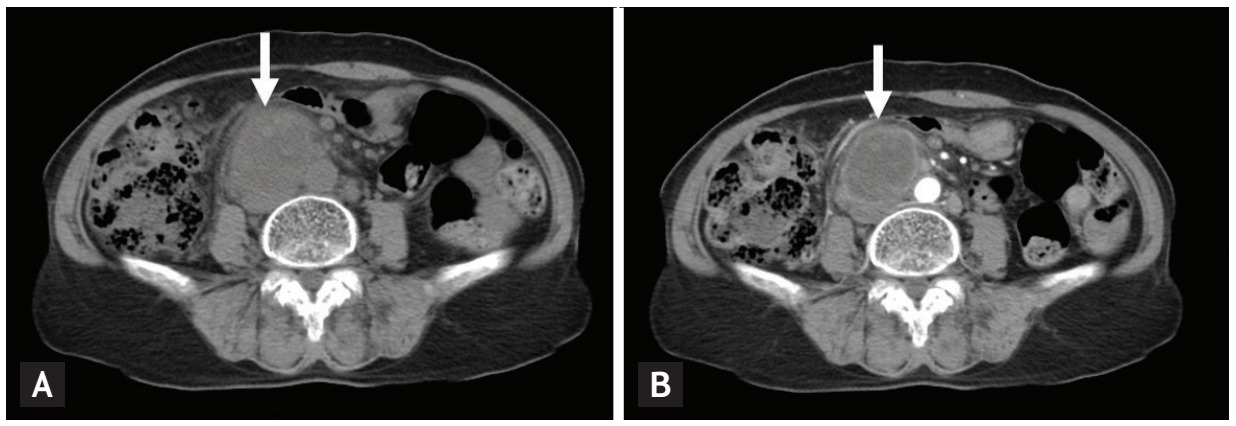

Figure 1. Computed tomography scans of the abdominopelvic area (A, precontrast; B, postcontrast) revealed an enhancing mass with prominent necrotic change in the aortocaval space, which was about $7.4 \mathrm{~cm}$ in size (arrows). 

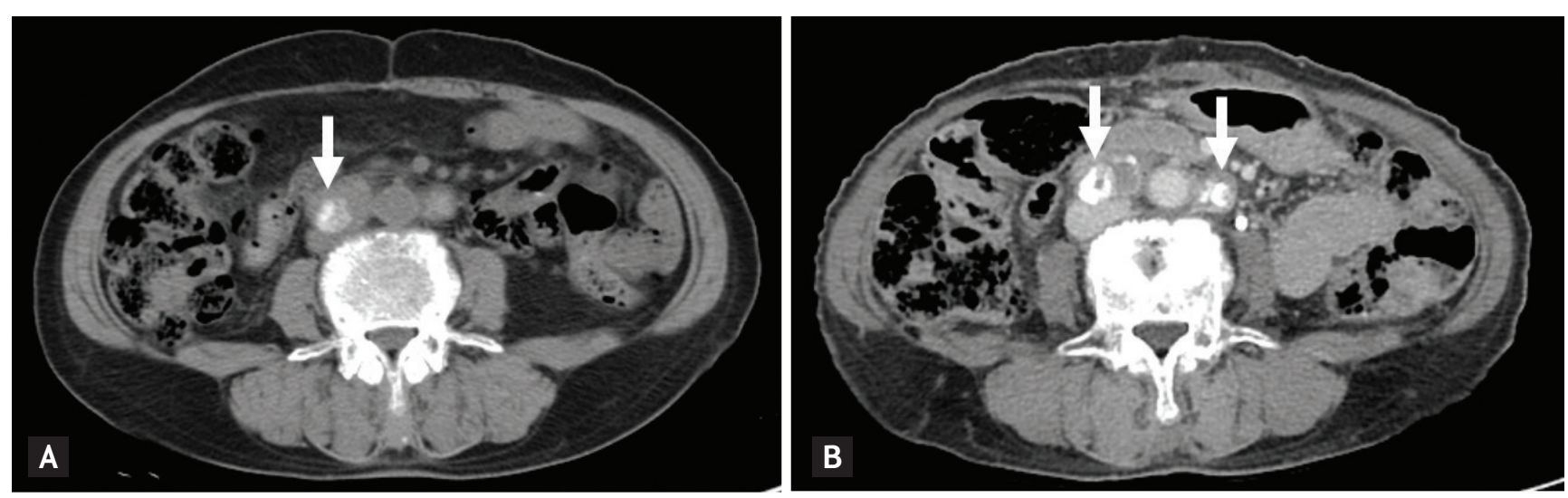

Figure 2. Computed tomography scan of the abdominopelvic area showed newly developed internal calcification in and around the aortocaval and left para-aortic areas, which was considered as dystrophic calcification during treatment (A, 8o weeks after treatment; B, 157 weeks after treatment) (arrows).

with disease progression, some types of malignancies such as gastrointestinal stromal tumor, non-Hodgkin's lymphoma, and Hodgkin's disease show response to treatment in the form of calcifications. Herein, we report a case of dystrophic calcification during palliative chemotherapy in a patient with recurrent renal cell car- cinoma who showed a long term response to treatment.

\section{Conflict of interest}

No potential conflict of interest relevant to this article was reported. 